\title{
MATTHIAS EMRICH
}

\section{Abschluss der Kritischen Friedrich-Schlegel-Ausgabe}

Der Abschluss der für die Romantikforschung zentralen Kritischen Friedrich-Schlegel-Ausgabe (KFSA) ist mit der Bewilligung eines entsprechenden Projektantrags durch die Deutsche Forschungsgemeinschaft in greifbare Nähe gerückt. Das an der Johannes Gutenberg-Universität Mainz angesiedelte, dort eng mit dem Forschungsschwerpunkt Historische Kulturwissenschaften assoziierte und von Ulrich Breuer (Deutsches Institut) geleitete Projekt hat im August 20 I 2 seine Arbeit aufgenommen. Während der ersten Projektphase sollen bis 20 I 5 von den noch ausstehenden sieben Bänden vier fertiggestellt werden. Im geplanten Folgeprojekt sollen bis 20 I 8 die restlichen drei Bände erarbeitet werden. ${ }^{1}$

Im Zentrum des Projektes steht der Briefwechsel von Friedrich und Dorothea Schlegel. Die Von- und An-Briefe des Ehepaars liegen unvollständig und verstreut in zahlreichen Einzelausgaben sowie in Brief- bzw. Werkausgaben exponierter Briefpartner wie Novalis oder Friedrich Schleiermacher vor. Ernst Behler hatte daher das ambitionierte Projekt verfolgt, sämtliche Von- und An-Briefe Friedrich und Dorothea Schlegels in einer einheitlichen Edition zu präsentieren, die er als dritte Abteilung der KFSA anlegte (Briefe von und an Friedrich und Dorothea Schlegel). Fünf Bände dieser Abteilung sind bisher erschienen, fünf weitere stehen noch aus.

Im Einzelnen handelt es sich um die Bände 26, 27, 28 sowie 3 I und 32. Band 26, der den Briefwechsel der Pariser und Kölner Zeit umfasst, wird von Hans Dierkes erarbeitet, der innerhalb der KFSA bereits die Kölner Vorlesungen Friedrich Schlegels ediert hat (Band I 5.2). Aufgrund der zahlreichen neu aufgefundenen Briefe aus dieser Zeit, die im Zuge einer von der Fritz Thyssen-Stiftung sowie von der Förderlinie I der Johannes Gutenberg-Universität Mainz unterstützten systematischen Suche (incl. Digitalisierung) von Brie-

1 Vgl. auch den Bericht von Ulrich Breuer/Till Dembeck/Maren Jäger: »Zum Stand der Kritischen Friedrich-Schlegel-Ausgabe«. In: Athenäum I 8 (2008), S. $165-182$. 
140 fen von und an Friedrich und Dorothea Schlegel entdeckt und gesichert worden sind, zeichnet sich für den mehr als 450 Briefe umfassenden Band ein erheblicher Umfang ab. Die Briefe stammen aus dem Zeitraum zwischen Mai I 802 und Juni I 808. In diesem Zeitraum fand bei Friedrich und Dorothea Schlegel eine in mehrfacher Hinsicht symptomatische Entwicklung statt, die in der Konversion zur katholischen Kirche im April i 808 ihren Kulminationspunkt gefunden hat. Auch Friedrich Schlegels Fokussierung auf Europa, seine literaturgeschichtlichen und indologischen Studien sowie die Kölner Vorlesungen zur Entwicklung der Philosophie in zwölf Bänden, zur Universalgeschichte und Über deutsche Sprache und Literatur fallen in diesen Zeitraum.

Die Bände 27 und 28, die die Korrespondenz der ersten Wiener Jahre umfassen werden ( I 808-I 8 I 4), betreut Barbara Otto (Wien/ Mainz). Sie hatte bereits zuvor im Rahmen des Thyssen-Projektes und mit Unterstützung der Johannes Gutenberg-Universität Mainz nach Schlegel-Autographen gesucht und war mit der Erstellung einer Datenbank, die sämtliche bekannten Briefe von und an Friedrich und Dorothea Schlegel systematisch erschließt, betraut. Dank einer Kooperation mit dem Institut für Kulturwissenschaften und Theatergeschichte der Österreichischen Akademie der Wissenschaften kann Frau Otto die anfallenden Recherchearbeiten und Quellenstudien in Wien, Prag und Budapest mit institutioneller Unterstützung vor Ort durchführen.

Die Korrespondenz der Wiener Jahre, vom Eintreffen Friedrich Schlegels in Wien am 22. Juni I 808 bis zum Wiener Kongress I 8 I 4 , wird hier erstmalig geschlossen präsentiert. Auch Dorotheas Briefwechsel aus dieser Zeit wird erstmals in authentischer Vollständigkeit ediert. Historisch-biographisch reflektieren diese Briefe die Zeitspanne von der Anstellung Friedrich Schlegels als Hofsekretär der Armeehofkommission ( I 809) im Feldzug gegen Napoleon im Stab des Erzherzogs Karl, seine Redakteurstätigkeiten bei der Armeezeitung, der Österreichischen Zeitung ( I 809) sowie bei dem Österreichischen Beobachter ( 18 I 0 ) im Dienst Metternichs bis zu seinen Wiener Vorlesungsreihen Über die neuere Geschichte ( I 8 I o) und Über die Geschichte der alten und neuen Literatur ( I 8 I 2 ) sowie schließlich die Gründung des Deutschen Museums ( 18 I 2 ). 
Seit 2008 hat es eine Reihe von Vorarbeiten gegeben, die dem nun 141 anlaufenden Projekt eine aussichtsreiche Ausgangsposition verschafft haben. So wurde an der Johannes Gutenberg-Universität Mainz eine Friedrich-Schlegel-Arbeitsstelle gegründet, die zunächst mit Maren Jäger besetzt war und nun mit Matthias Emrich besetzt ist. Mit Unterstützung von Barbara Otto wurde seitens der Arbeitsstelle eine Datenbank entwickelt, in der die durch systematische Suche zusammengetragenen und digitalisierten Briefe von und an Friedrich und Dorothea Schlegel mit sämtlichen relevanten Metadaten erfasst sind. Die Datenbank zählt derzeit etwa 3.000 Datensätze. Der überlieferte Briefbestand verteilt sich auf 79 Archive und Bibliotheken, wobei sich 28 Institutionen im Ausland befinden (u. a. Estland, Frankreich, Schweden, Polen, Ungarn, USA). Die Arbeitsgrundlage der Editionsarbeiten bilden die bisher beschafften digitalen Faksimiles. Es liegen bislang etwa 4.500 Digitalisate vor, die aus über 40 Archiven stammen. Die inhaltliche Auswertung und systematische Abgleichung ist noch nicht abgeschlossen und verspricht bislang unbekannte Quellenfunde.

Das Vorhaben Ernst Behlers, sämtliche Werke und Briefe Friedrich Schlegels kritisch zu edieren, verdient trotz der Kritik an den unter seiner Ägide erschienenen Bänden Respekt. Behlers Vorhaben lässt sich nur dann zum Abschluss bringen, wenn an seine editorischen Entscheidungen zumindest partiell angeschlossen wird. Allerdings geht Behlers Konzept auf einen Stand der Editionswissenschaft zurück, der heute veraltet ist; der erste Band der KFSA erschien 1958. Es war daher unerlässlich, für die ausstehenden Bände neue editorische Richtlinien zu erstellen. Dazu gehört, dass die Verweistechnik, die bislang für nahezu jeden Band individuell angepasst wurde, standardisiert werden wird. Text- und Kommentar-Teil werden funktional getrennt. Die Textkonstitution folgt der Prämisse, einen zitierbaren Text zu präsentieren, bleibt dabei jedoch dem Prinzip der Authentizität streng verpflichtet. So wird der kritische Apparat, der sämtliche Korrekturen Schlegels (Varianten), aber auch allenfalls notwendige Texteingriffe des Herausgebers (Emendationen) verzeichnet, jedem Brief unmittelbar beigegeben. Der Kommentarteil bietet die weiterführende Dokumentation der handschriftlichen und gedruckten Überlieferung (Zeugenbeschrei- 
142 bung, Erstdrucke) und textbegleitende Einzelstellen-Erläuterungen, die dem heutigen Leser sämtliche sonst verdeckten historischen Bedeutungsschichten erschließen.

Neben den Briefbänden stehen noch zwei weitere Bände der KFSA aus. In einem dritten (und letzten) Teilband zu Band i 5 werden innerhalb der zweiten, den Nachlass Friedrich Schlegels umfassenden, Abteilung drei Textkomplexe zur antiken Poesie und Philosophie präsentiert: Die sogenannten >Leipziger Manuskripte<, die im Wesentlichen aus den Handschriften der beiden frühen Aufsätze Von den Schulen der Griechischen Poesie und Vom aesthetischen Werth der Griechischen Komödie bestehen, sowie die Notizhefte Studien des Alterthums und Fragmente zur Geschichte der Griechischen Poesie. Band I 5.3 wird kooperativ von einem Schlegel-Spezialisten (Armin Erlinghagen, Bad Münstereifel) und einem Altphilologen (Thomas Schirren, Salzburg) herausgegeben.

Band 34, der einzige noch innerhalb der vierten Abteilung ausstehende Band, wird von Christian Benne (Odense) bearbeitet und widmet sich Schlegel als Herausgeber. Im Zentrum wird Schlegels Lessing-Edition stehen, wobei neben den editorischen Paratexten auch die von ihnen gerahmten Texte Lessings stehen werden, um das von Schlegel intendierte, authentisch-vollständige Textensemble lesbar zu machen. Die rezeptionsvermittelnden Schriften Schlegels, die im größeren Kontext kulturpolitisch motivierter Quellenerschließung während der mittleren Phase der Romantik stehen, gehören zu den Desideraten der Schlegel-Forschung. Einleitungen, die Friedrich Schlegel als Herausgeber der Schriften seiner Frau verfasste, werden den Band ergänzen. Übersetzungen, die von Dorothea geleistet wurden, sollen im Kommentar ausführlich dokumentiert werden.

Die Editionsergebnisse werden als TEI-P5 konforme XMLFormate kodiert. Damit ist für das hybrid angelegte Projekt eine Ausgabeneutralität der gewonnen Daten sichergestellt. Nach der Fertigstellung der gedruckten Bände stehen die Daten für eine Online-Publikation in einem Friedrich-Schlegel-Portal offen. Gleichzeitig bietet das Projekt damit verwandten Vorhaben ein erhebliches Anknüpfungspotential, etwa der digitalen Edition der Briefe August Wilhelm Schlegels, oder auch möglichen kooperativen For- 
schungsanwendungen, beispielsweise einer digitalen Rekonstruk- 143 tion von Briefnetzwerken um I 800 . Für die informatischen Dimensionen des Projekts konnte das Trier Centre for Digital Humanities als Kooperationspartner gewonnen werden. ${ }^{2}$

Fragen, Anregungen, nicht zuletzt aber Informationen zu mutmaßlich noch unbekannten Schriften Friedrich Schlegels und Briefen von und an Friedrich und Dorothea Schlegel werden erbeten an den Mainzer Projektkoordinator (matthias.emrich@uni-mainz.de) oder an den Projektleiter (ulrich.breuer@uni-mainz.de).

2 Weitere Informationen unter http://www.uni-mainz.de/kfsa.

Abschluss der Kritischen Friedrich-Schlegel-Ausgabe 